\title{
Detection for Multisatellite Downlink Signal Based on Generative Adversarial Neural Network
}

\author{
Qing-yang Guan (iD) ${ }^{1,2}$ and Wu Shuang ${ }^{1}$ \\ ${ }^{1}$ College of Engineering, Xi'an International University, Xi'an 710077, China \\ ${ }^{2}$ College of Electronic and Information Engineering, Shenyang Aerospace University, Shenyang 110136, China \\ Correspondence should be addressed to Qing-yang Guan; gqy_gqy@163.com
}

Received 31 May 2020; Revised 11 July 2020; Accepted 23 July 2020; Published 12 August 2020

Guest Editor: Jun Shen

Copyright (C) 2020 Qing-yang Guan and Wu Shuang. This is an open access article distributed under the Creative Commons Attribution License, which permits unrestricted use, distribution, and reproduction in any medium, provided the original work is properly cited.

\begin{abstract}
A method for satellite downlink signal detection based on a generative adversarial network is proposed. The generator adversarial network and adversarial network are established, respectively. The generator network realizes the local generator of satellite signals, and the adversarial network is used for high-precision signal detection. The error network is generated by the error signal to form the satellite link downlink. The network reconstructs the optimal weights by generating errors, forms an error matrix for different satellite downlink, and then forms an adaptive matrix weight adjustment. Through the reconstruction of the optimal detection matrix, detection for the downlink signals of multiple satellites is completed. The proposed generative adversarial network can realize the high-precision detection for the downlink signal.
\end{abstract}

\section{Introduction}

1.1. Background Introduction. Due to the rapid development of AI technology, technologies are used in signal detection of the satellite to ground links. For the typical satellite to ground downlink, due to the low-orbit satellite, the relatively large-scale movement introduces a Doppler frequency shift. Doppler frequency will occur between the users and the loworbit satellites. Downlink for low-orbit satellite mobile communication system is sensitive to carrier frequency offset, which seriously destroys the orthogonality between subcarriers and causes distortion to the receiver. A broadband signal detection technology is developed from AI technology. AI technology based on deep learning is applied in various fields, such as inserting and extracting knowledge [1], speech recognition [2], and language translation. Since deep learning has sufficient hidden layers, it can accurately simulate the target function and optimization direction [3]. In $[4,5]$, sparse coding is applied for data detection, including spectrum analysis of communication systems [6] and channel estimation [7]. Sparse coding has the following two important features, which are suitable for selective frequency channel and multiuser detection. Chen et al. [8] established a deep neural network, which uses fewer data to complete labeling and training and then optimizes the deep neural network by reverse tuning.

For communication systems, Gaur and Ingram [9] proposed a simple MMSE Interference Suppression for Real and Rate-1/2 Complex Orthogonal Space-time Block Codes. In [10-13], the multiuser detection methods were proposed, especially for massive MIMO systems. In [14, 15], data encoding method was proposed, which improved accuracy through precoding. Çelebi and H. Arslan [16] proposed the ML-SIC receiver for the theoretical analysis of the coexistence of the LTE-A system. Rusek et al. [17] proposed a broadband mass MU-MIMO detection based on the ZF equalization algorithm, which uses interpolation to improve accuracy. But it requires large-scale matrix operations to increase the complexity. Ahn et al. [18] proposed the Sparsity-Aware Ordered Successive Interference Cancellation for Massive Machine-Type Communications.

For the research of deep learning neural networks, Ghamisi et al. [19] proposed a classification algorithm to extract features and classify data. Then, the algorithm forms 
classification templates to improve classification accuracy. A kernel-space algorithm is proposed in [20], which establishes a transformed network in order to further improve the data classification method.

Yuan et al. [21] combined sparse coding and Markov random fields to establish the classification network based on spatial data correlation, which is to improve classification accuracy. On this basis, Wang et al. [22] propose the subspace analysis method to further the accurate classification performance of the network.

For data classification and detection, the above methods do not use deep networks. Bengio et al. [23] proved that only classification methods such as SVM or logistic regression cannot effectively improve classification, such as decision trees or kernel-space transformation. The single-layer classification method could not obtain better classification accuracy. Depth models, including multiple hidden layers, are identified through acquiring target data features. Deep learning models are widely used in related research fields, such as image classification and speech recognition $[2,24]$. Zhang et al. [25] also gave a summary analysis of deep learning. In 2014, a deep learning architecture based on SAE encoding was proposed for data classification [26]. Later, a deep learning architecture based on DBN was used for HSI data classification [27, 28].

Deep learning networks, such as deep CNN networks [29], were used in image recognition and classification. $\mathrm{Li}$ et al. [30] proposed a novel pixel-pair method for the classifier, which could use less training data and label data. A deep learning architecture based on CNN was proposed in $[31,32]$. For example, Yue et al. [32] proposed a PCA-based analysis method. In [33, 34], deep learning architecture was proposed, combined with sparse coding and Gabor filter for deep feature extraction. On this basis, authors in $[35,36]$ proposed to use the CNN architecture deep learning network for classification and recognition for hyperspectral imagery.

1.2. Reasons for Proposed Algorithm. The biggest advantage of deep learning is that it can realize the complex nonlinear function approximation of massive data through nonlinear network architecture, then characterize the distribution, and form the ability to learn the essence of data features.

If the features of the data change or the types of data expand, the ability for deep learning describing massive data becomes weaker. For multisatellite downlink signal detection, the multisatellite downlink channel variety is complex. For multisatellite downlink service scenarios, the model established by deep learning is not universal, and the model of each communication type changes. So the number of variables that deep learning can provide is limited, and the number of layers of the deep network is also limited.

Secondly, deep learning requires excessively high-quality training data. The accuracy of data analysis increases as the training data increase. In satellite downlink, high-quality training data cannot be obtained in many communication scenarios, so poor quality data could not be formed for deep learning to obtain a general effective model.
How to use a small amount of training data and establish reliable multisatellite downlink detection in different scenarios is important. In this paper, we use the GAN network to overcome this difficulty in satellite downlink. Zhang et al. [37] proposed a GAN network based on game theory, which consists of the generator network and the discriminator network. The generator network is used to generate data to improve the recognition accuracy, and the discriminator network is used to distinguish the target. Wang et al. [38] adopted the BP method to train the generating network and the discriminating network separately.

GAN network is a model that includes the $G$ generator network part and the D network part [39]. The generator network and the discriminator network part are formed in an adversarial manner. Given the advantages of adversarial networks, Chen et al. [40] applied adversarial networks to image data. Creswell et al. [41] applied GAN network to data analysis, feature extraction, and classification.

In order to improve the performance of the GAN network, in [42, 43], improved modes were proposed. These improved modes are divided into two categories, one based on structural optimization and the other based on objective optimization. Sun et al. [42] proposed the generative adversarial network, which introduced variables to improve the efficiency of GAN games. Based on generative networks, in addition, Yu et al. [43] proposed conditional information adversarial networks based on mutual information to improve the efficiency of generating networks.

In the optimization process, in [40, 44-46], the coding part for the GAN network was added. The coding part could improve the accuracy and the efficiency as the whole and improve the GAN network objective function optimization by adding a label classifier. In order to improve the convergence of the GAN network, in [47-51], an optimized objective function was proposed to improve the training process of the GAN network. Among them, authors in [47-49] used different models to achieve the loss objective function.

For multisatellite downlink signal detection, highquality training data cannot be obtained in many downlink scenarios. The models established by traditional deep learning are not universal, so the model of the downlink data type varies with different channel models. But deep learning requires too much quality for training data. Therefore, we propose the GAN network. The main goal is using few data training information to form through adversarial generation network under poor channel conditions and then to achieve efficient and high-precision signal detection.

\section{System Model and Problem Formulation}

2.1. System for Satellite Downlink. Figure 1 shows the multisatellite downlink transmission network. The satellite network is divided into the space segment and the ground segment. The space segment is composed of multiple satellites, and the ground segment is composed of ground users. The space segment communicates with the ground segment through the downlink channel. 


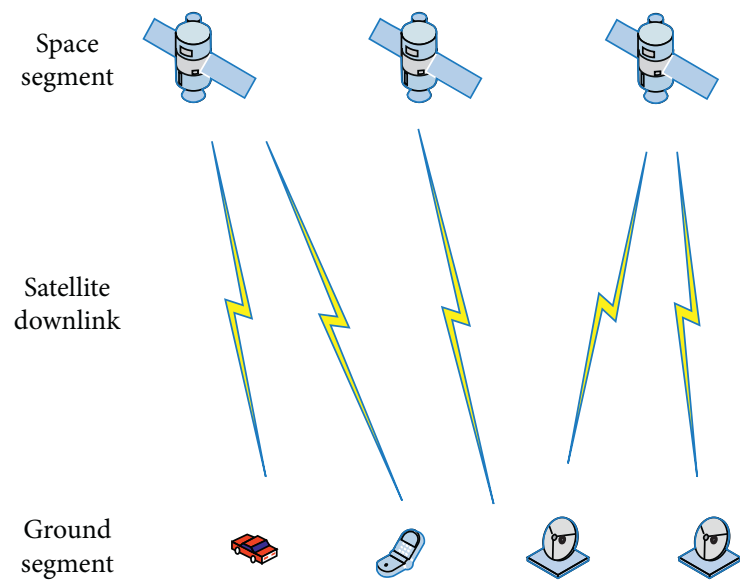

Figure 1: Diagram for multisatellite downlink transmission network.

Due to the high-speed movement, define multipath propagation delay as $\tau$. The time channel impulse response function for frequency-selective mobile satellite channel is $h(t, \tau)$. For different $\tau, h(t, \tau)$ is not related to each other. For definite delay $\tau, h(t, \tau)$ is an average complex Gaussian random process. Simultaneously, impulse response $h(t, \tau)$ is according to the flat fading characteristics. Therefore, the time-varying impulse response of $L$ multichannel can be expressed as follows:

$$
h(t, \tau)=\sum_{l=0}^{L-1} b_{l} v_{l}(t) \delta\left(\tau-\tau_{l}\right)
$$

where $\tau_{l}$ is the transmission delay of the $l$ th path. $v_{l}(t)$ is the complex Gaussian process, which is the weight component of the $l$ th path; the power spectrum is the Doppler power spectrum of the $l$ th path, which also can represent the fading rate of the $l$ th path. $b_{l}$ is the time delay coefficient, and the value is the square root of the average delay power for the $l$ th path, which can be expressed as the weighting of unresolved paths arriving at different incidence angles within a certain delay interval.

$d(t)$ and $r(t)$ represent the system input and output, respectively, so that the channel can be expressed as follows:

$$
r(t)=\sum_{l=0}^{L-1} b_{l} v_{l}(t) d\left(\tau-\tau_{l}\right)
$$

The amplitude $b_{0}(t)$ of the direct path can be considered as Rician distribution, while the amplitude of other paths can be considered as $b_{l}(t)$, and $l=1,2,3, \ldots, L-1$ can be considered as Rayleigh distribution. The downlink satellite channel simulation model proposed in this paper is essentially based on Rician and Rayleigh fading signals with specific Doppler spectrum. Simulation for Rician and Rayleigh processes requires two Gaussian processes. Sinusoidal superposition is generally used to simulate these Gaussian processes.

$v_{l}(t)$ is defined as the complex Gaussian process, which is satisfied as follows:

$$
v_{l}(t)=v_{r, l}(t)+j v_{i, l}(t),
$$

where $v_{r, l}(t)$ is the real part and $v_{i, l}(t)$ is the imaginary part, which are independent of each other and have the same mean and autocorrelation function. $v_{i, l}(t)$ can be expressed as follows:

$$
v_{i, l}(t)=\sum_{n=1}^{K_{i, l}} c_{i, n, l} \cos \left(2 \pi f_{i, n, l} t+\theta_{i, n, l}\right),
$$

where $K_{i, l}$ is the number of sine waves of the $l$ th path, $f_{i, n, l}$ is the $n$th Doppler shift of the $l$ th path, $\theta_{i, n, l}$ is the $n$th Doppler phase of the lth path, and $c_{i, n, l}$ is the $n$th Doppler coefficient of the lth path.

$\theta_{i, n, l}$ is uniformly distributed within the interval $[0,2 \pi)$; get it by taking the random number in. $c_{i, n, l}$ and $f_{i, n, l}$ can be calculated using the MEA method and the Monte Carlo method.

In the tapped delay line method to establish a channel model of a satellite-ground link, the multipath channel impulse response is composed of multiple paths with different delay characteristics, and each path has specific signal amplitude fading and power spectrum characteristics. The proposed model is measured with broadband satellite downlink channels in the wilderness, rural, and urban environments, with the signal carrier frequency of $1.02 \mathrm{GHz}$. Tables 1-3 show the measured channel characteristic parameters of the wilderness environment, rural environment, and urban environment, respectively.

Figures 2-7 show the signal amplitude and Doppler power spectrum for each tap of the direct component for satellite mobile channel in the wilderness environment, rural environment, and urban environment, respectively, established by using tapped delay lines.

Due to different satellite simulation, multipath delays of the channels are different, and the signal amplitude fading is completely different. The first path of each simulation scenario has a large impulse response corresponding to the amplitude. This is because the first path has a direct component, and its envelope corresponds to the Rician channel density distribution. For rural simulation scenarios, the reflection of signals from more buildings and the diffraction effect cause a larger number of multipaths, and the extended delay of the received signal is larger, which makes the signal get more severe fading. For the rural simulation scene, compared with the urban scene, the number of buildings is small. The reflection and refraction phenomena are reduced compared to the urban simulation environment, so the number of multipaths is reduced, and the channel fading is flat compared to the urban environment. For urban simulation, because the number of buildings is smaller than the number of urban simulation, and the number of vegetation is reduced compared to the rural environment, the reflection and refraction phenomena are reduced compared to urban and rural areas, and channel fading is also more difficult than urban environments. 
TABLE 1: Channel model parameter in the wilderness environment.

\begin{tabular}{|c|c|c|c|c|c|}
\hline Tap & Distribution function & Parameter & Parameter distribution & Value $(\mathrm{dB})$ & Delay (ns) \\
\hline 1 & $\begin{array}{c}\text { LOS Rician } \\
\text { NLOS Rayleigh }\end{array}$ & $\begin{array}{c}\text { Rician factor } \\
\text { Average multipath power }\end{array}$ & $\begin{array}{c}K \\
2 \sigma_{1}^{2}\end{array}$ & $\begin{array}{c}6.3 \\
-9.5\end{array}$ & 0 \\
\hline 2 & Rayleigh & Average multipath power & $2 \sigma_{l}^{2}$ & -24.1 & 100 \\
\hline
\end{tabular}

TABLE 2: Channel model parameter in the rural environment.

\begin{tabular}{|c|c|c|c|c|c|}
\hline Tap & Distribution function & Parameter & Parameter distribution & Value $(\mathrm{dB})$ & Delay (ns) \\
\hline \multirow{2}{*}{1} & LOS Rician & Rice factor & $K$ & 5.3 & \multirow{2}{*}{0} \\
\hline & NLOS Rayleigh & Average multipath power & $2 \sigma_{l}^{2}$ & -12.1 & \\
\hline 2 & Rayleigh & Average multipath power & $2 \sigma_{l}^{2}$ & -17.0 & 60 \\
\hline 3 & Rayleigh & Average multipath power & $2 \sigma_{l}^{2}$ & -18.3 & 100 \\
\hline 4 & Rayleigh & Average multipath power & $2 \sigma_{l}^{2}$ & -19.1 & 130 \\
\hline 5 & Rayleigh & Average multipath power & $2 \sigma_{l}^{2}$ & -22.1 & 250 \\
\hline
\end{tabular}

TABLE 3: Channel model parameter in the urban environment.

\begin{tabular}{|c|c|c|c|c|c|}
\hline Tap & Distribution function & Parameter & Parameter distribution & Value $(\mathrm{dB})$ & Delay (ns) \\
\hline \multirow{2}{*}{1} & LOS Rician & Rician factor & $K$ & 9.7 & \multirow{2}{*}{0} \\
\hline & NLOS Rayleigh & Average multipath power & $2 \sigma_{l}^{2}$ & -7.3 & \\
\hline 2 & Rayleigh & Average multipath power & $2 \sigma_{l}^{2}$ & -17.6 & 30 \\
\hline 3 & Rayleigh & Average multipath power & $2 \sigma_{l}^{2}$ & -18.3 & 180 \\
\hline 4 & Rayleigh & Average multipath power & $2 \sigma_{l}^{2}$ & -19.3 & 60 \\
\hline 5 & Rayleigh & Average multipath power & $2 \sigma_{l}^{2}$ & -22.1 & 100 \\
\hline 6 & Rayleigh & Average multipath power & $2 \sigma_{l}^{2}$ & -25.3 & 190 \\
\hline 7 & Rayleigh & Average multipath power & $2 \sigma_{l}^{2}$ & -28.1 & 250 \\
\hline 8 & Rayleigh & Average multipath power & $2 \sigma_{l}^{2}$ & -29.1 & 270 \\
\hline
\end{tabular}

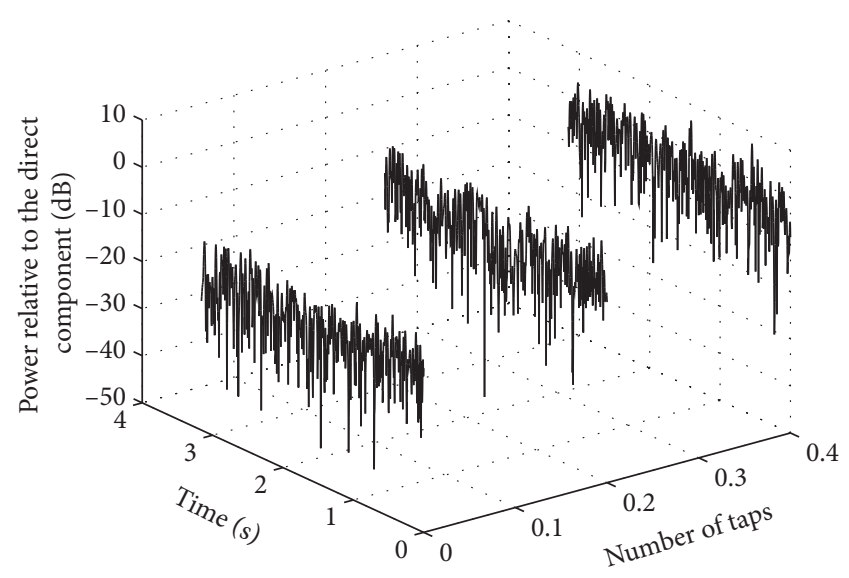

FIgURE 2: Signal amplitude for each tap (wilderness environment).

2.2. Problem Formulation for Detection of Satellite Downlink. Figure 8 shows a block diagram of the multisatellite for the downlink communication system. The subcarrier allocation method uses IFDMA; after the user obtains the carrier allocation, the subcarriers of each user do not overlap with each other. And the satellite receives superimposed interference. It is assumed that the signals of each user can be separated by the carrier mapping of the signal. Due to the high-speed movement, the carrier frequency offset is introduced into the transmission signal, and its value depends on the speed of the satellite and the maximum elevation angle.

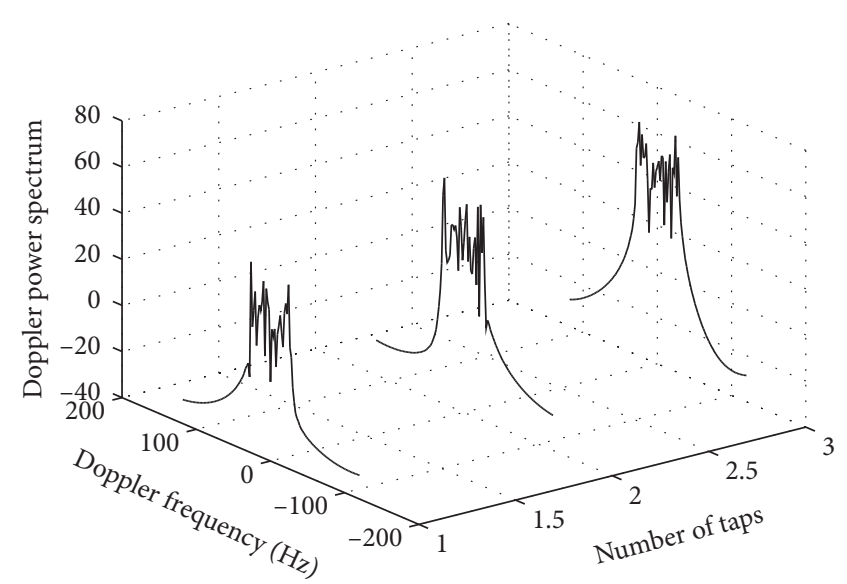

FIGURE 3: Doppler power spectrum for each tap (wilderness environment).

Because of receiving multiple satellite signals, the relative frequency offset factors of satellites are also different. When the number of system subcarriers is determined, access interference will be introduced due to differences of multiple satellites. Figure 8 shows a block diagram of multisatellite for the downlink DFT-S OFDM system. The subcarrier allocation method uses IFDMA; after allocating the carrier, the subcarriers of each user do not overlap with each other. The satellite receives each user with overlapping interference. It is assumed that the multisatellite signal can be separated by the carrier mapping of the signal. 
Define the relative frequency offset factor $\xi^{i}$ of the $i$ th satellite, and the number of satellites received by the downlink ground user is $N$. The frequency domain signal is modulated by $N$ orthogonal subcarriers, and the time domain signal of multiple satellites received by the ground user can be expressed as follows:

$$
\begin{aligned}
y_{i}(m)= & \frac{1}{N} \sum_{k=0}^{N-1} S(k) \sum_{l=0}^{L-1} h(m, l) \exp \left(j 2 \pi k \frac{(m-l)}{N}\right) \\
& \cdot \exp \left(j 2 \pi \xi^{i} \frac{m}{N}\right)+z(k),
\end{aligned}
$$

where $z(k)$ is. The receiver performs $\mathrm{N}$-point modulation conversion; it can be obtained as

$$
\begin{aligned}
Y_{i}\left(k^{\prime}\right)= & \frac{1}{N} \sum_{k=0}^{N-1} \sum_{m=0}^{N-1} S(k) H(k) \exp \left(j 2 \pi m \frac{k}{N}\right) \\
& \cdot \exp \left(j 2 \pi \xi \frac{m}{N}\right) \cdot \exp \left(-j 2 \pi m \frac{k^{\prime}}{N}\right)+Z\left(k^{\prime}\right),
\end{aligned}
$$

where the ground user receives the signal of the $i$ th satellite with interference $Y_{i}\left(k^{\prime}\right)$, which can be obtained by separating the interference items:

$$
\begin{aligned}
Y_{i}\left(k^{\prime}\right)= & \frac{1}{N} \sum_{k=0}^{N-1} \sum_{m=0}^{N-1} S(k) H(k) \exp \left(j 2 \pi m \frac{k}{N}\right) \exp \left(j 2 \pi \xi^{\prime} \frac{m}{N}\right) \\
& \cdot \exp \left(-j 2 \pi m \frac{k^{\prime}}{N}\right)+Z\left(k^{\prime}\right), \\
= & \frac{1}{N} \sum_{m=0}^{N-1} \sum_{k=0}^{N-1} S\left(k^{\prime}\right) H\left(k^{\prime}\right) \exp \left(j 2 \pi \xi^{m} \frac{m}{N}\right) \\
& +\sum_{m=0}^{N-1} \sum_{k=0,}^{N-1} X(k) H(k) \exp \left(j 2 \pi m \frac{\left(k-k^{\prime}\right)}{N}\right) \\
& \cdot \exp \left(j 2 \pi \xi^{\prime} \frac{m}{N}\right)+Z\left(k^{\prime}\right), \quad k^{\prime}=0,1, \ldots, N-1 .
\end{aligned}
$$

The first term is the interference between communication symbols, the second term is the interference introduced by communication access, and the third term is the interference introduced by Gaussian white noise. The multiple satellite signals received by the downlink ground user can be expressed as follows:

$$
\begin{aligned}
& Y(k \prime)=\frac{1}{N} \sum_{i=1}^{T-1} \sum_{m=0}^{N-1} \sum_{k=0}^{N-1} S(k) H(k) \exp \left(j 2 \pi m \frac{k}{N}\right) \exp \left(j 2 \pi \xi^{i} \frac{m}{N}\right) \cdot \exp \left(-j 2 \pi m \frac{k^{\prime}}{N}\right)+Z(k \prime) \\
& =\frac{1}{N} \sum_{m=0}^{N-1} \sum_{k=0}^{N-1} S\left(k^{\prime}\right) H\left(k^{\prime}\right) \exp \left(j 2 \pi \xi^{i} \frac{m}{N}\right)+\sum_{\substack{i \in T \\
j \neq i}} \sum_{\substack{m=0 \\
j \neq i}}^{N-1} \sum_{\substack{k=0, k \neq k^{\prime}}}^{N-1} S(k) H(k) \exp \left(j 2 \pi m \frac{\left(k-k^{\prime}\right)}{N}\right) \exp \left(j 2 \pi \xi^{i} \frac{m}{N}\right) \\
& +\sum_{\substack{j \in T \\
j \neq i}} \sum_{m=0}^{N-1} \sum_{\substack{k=0, l \neq k^{\prime}}}^{N-1} X(l) H(l) \exp \left(j 2 \pi m \frac{\left(l-k^{\prime}\right)}{N}\right) \exp \left(j 2 \pi \xi^{j} \frac{m}{N}\right)+Z\left(k^{\prime}\right), \quad k^{\prime}=0,1, \ldots, M-1,
\end{aligned}
$$

where $Y\left(k^{\prime}\right)$ is the satellite signals received by ground users. The last term in the above formula is the reception interference introduced by receiving multiple satellites.

\section{Generative Adversarial Network Algorithm for Satellite Signal Detection}

3.1. Architecture of GAN Network for Satellite Signal Detection. Figure 9 gives the information processing flow based on the GAN algorithm. The generator network realizes the local signals, and the discriminator network is used for signal detection.
We define the generalized loss function model as follows:

$$
\begin{array}{ll}
\min & \|Y(k)-W S(k)\|_{F}^{2}+\alpha f(W), \\
\text { s.t. } & \left\|W_{j}\right\|_{2}^{2} \leq 1, \forall j,
\end{array}
$$

with adversarial network generator; we could achieve to obtain $\alpha f(W)$.

In order to obtain the signal detection of the satellite downlink, we implement the optimal weight $W$ by introducing the GAN network. The following discussion is aimed at using the adversarial network to obtain the optimal 


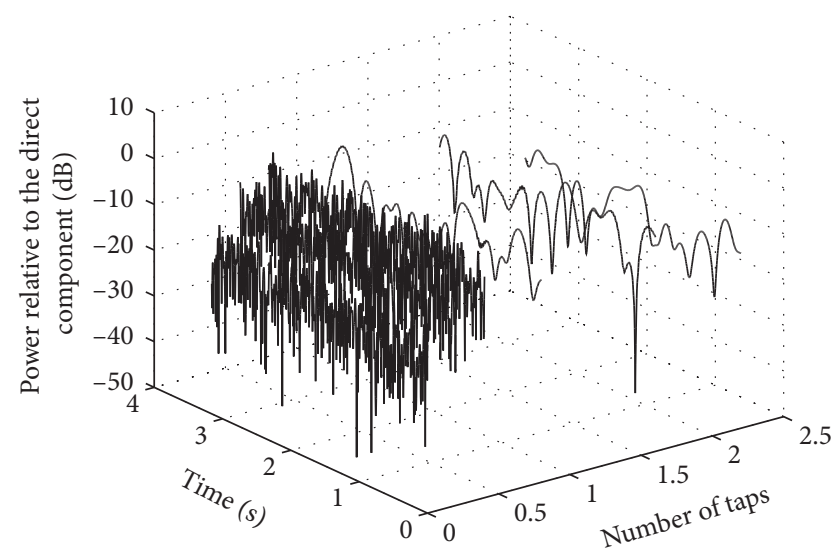

FIgURE 4: Signal amplitude for each tap (rural environment).

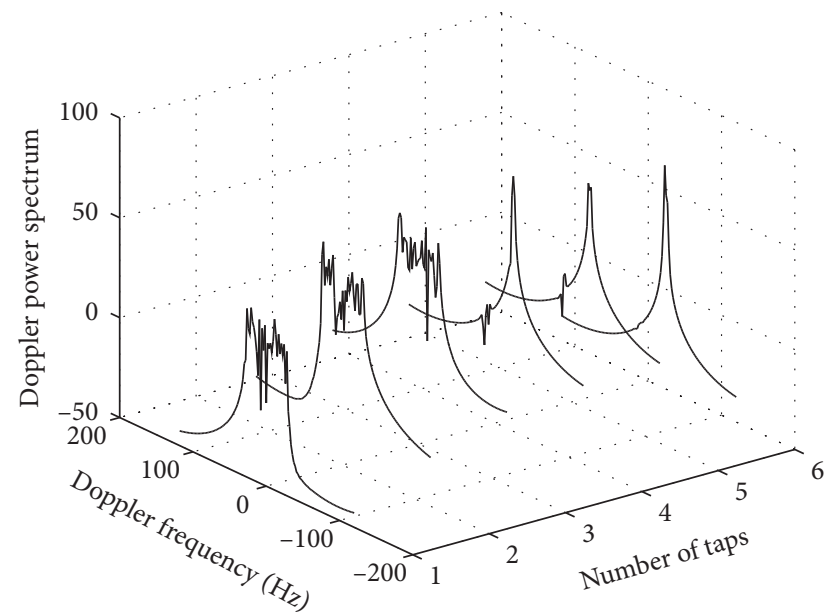

Figure 5: Doppler power spectrum for each tap (rural environment).

elimination matrix. In order to achieve optimal weights, we established the loss function of GAN network.

We introduce a generative adversarial network loss cost function to identify multisatellite downlink data. The cost function of the GAN can be expressed as follows:

$$
L_{\mathrm{GAN}}=E_{x \sim p_{t}}[\log D(s)]+E_{x \sim p_{t}}[\log (1-D(G(s)))] .
$$

From formula (10), we could obtain GAN which consists of a generator network and a discriminator network, where $G(s)$ is defined as generator network and $D(s)$ is defined as discriminator network.

The generator network $G(s)$ produces the output as the target domain as shown in Figure 10. However, combined with a wide range of capacity, the target is to minimize adversarial losses. It can ensure that the learning network is directed to the input $s$ which corresponds to the ideal output.

Figure 10 gives the adversarial network model for satellite signal processing flow. In order to further reduce the GAN function, we propose a reconstruction of generating error weights $W$. In order to reduce the number of iterations of reconstructed features, a method of closely

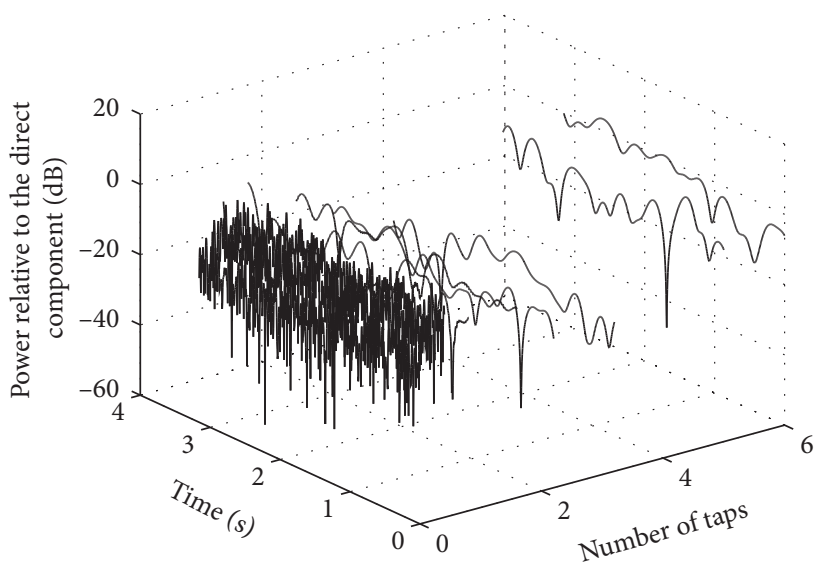

Figure 6: Signal amplitude for each tap (urban environment).

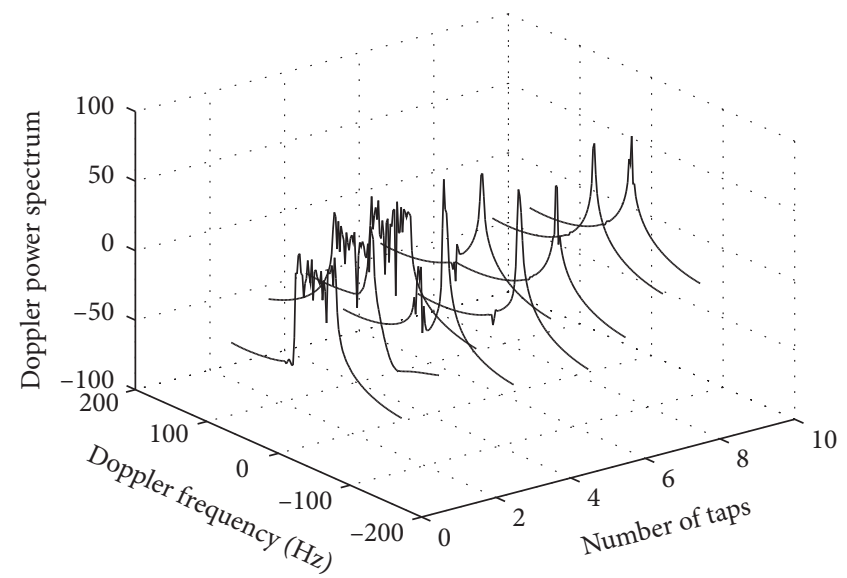

Figure 7: Doppler power spectrum for each tap (urban environment).

cost function is proposed. Then, we propose an iterative cost function $L_{\mathrm{GAN}}$ :

$$
L_{\mathrm{GAN}}=L_{G}+L_{D}
$$

where $L_{G}$ represents the cost function for the generator network. $L_{D}$ represents the cost function for the discriminator network. The generator network includes forward and backward features. That is to say, the separately generated network can be reconstructed according to the original generated data and then form bidirectional iteration.

Figure 11 gives the signal processing flow based on the adversarial network. From Figure 11, we also give the processing for GAN network establishment. The goal is to optimize the loss function, including two parts. That is $G$ network, and the second is the D network. In the following, we establish the generative network and the confrontation network separately.

3.2. Establishment for Generator Network. The process of generating regularization terms is as follows. Define $\left\{S_{i}, Y_{j}\right\}=\left\{\left(s_{1}, y_{1}\right),\left(s_{2}, y_{2}\right), \ldots,\left(s_{i}, y_{j}\right)\right\}$ as the generation set, where $s_{i}$ is the data feature after the $i$ th generation, $s_{j}$ is 


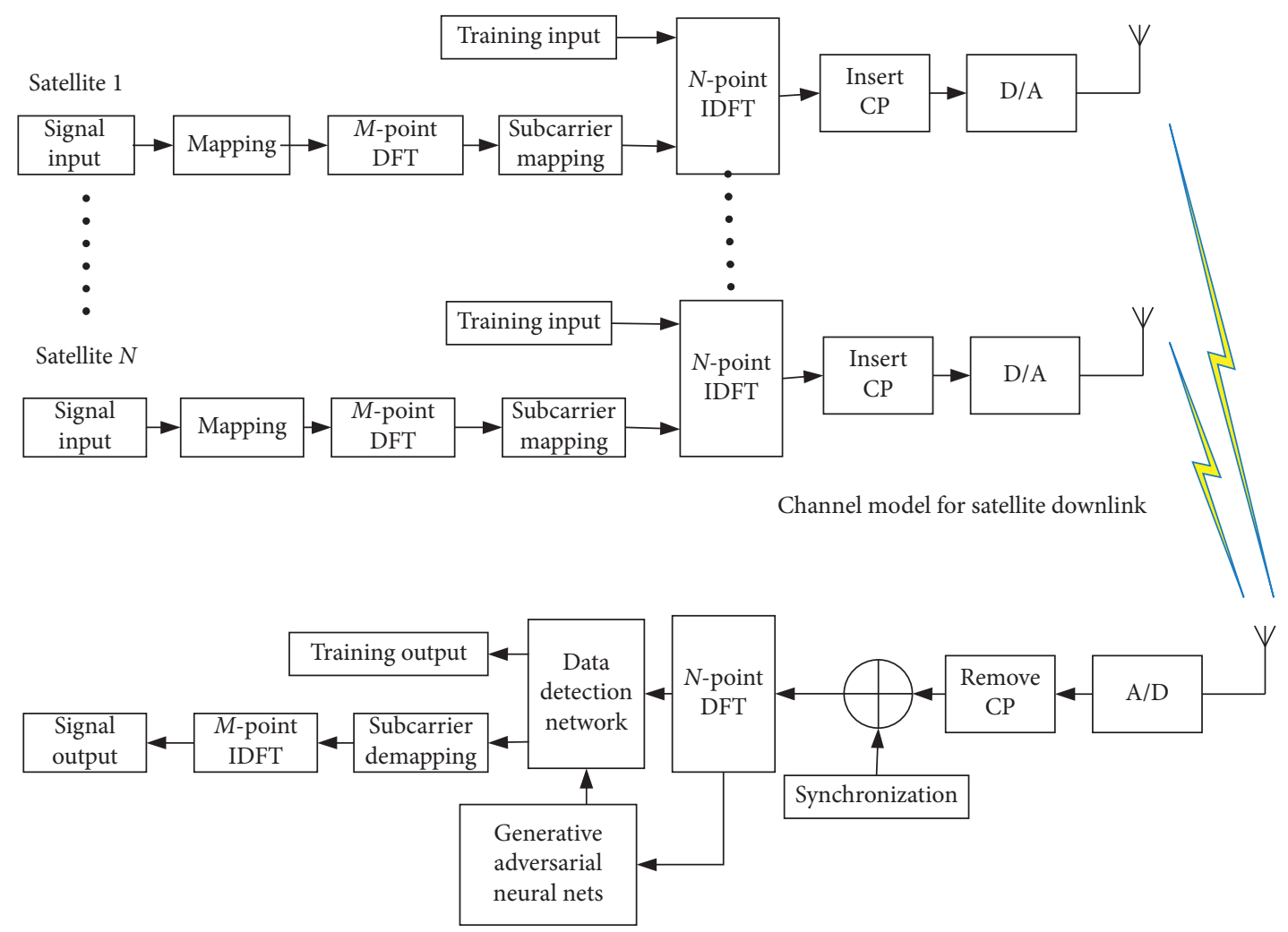

FIGURE 8: Multisatellite model for the downlink communication system.

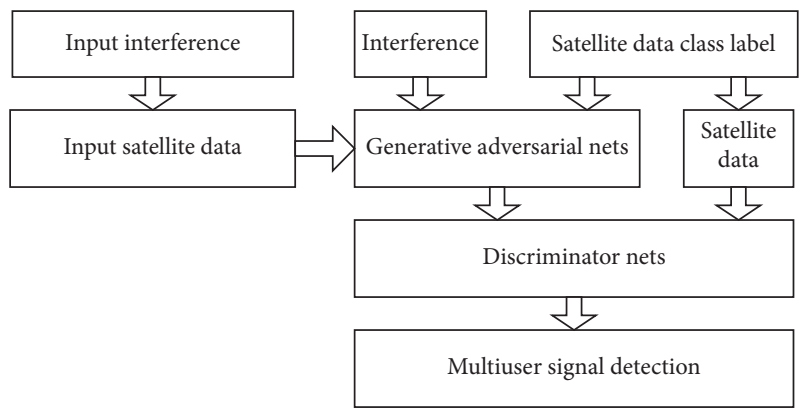

FIGURE 9: Information processing flow based on the GAN algorithm.

the data feature after the $j$ th generation, and $y$ is the expected detection data. We establish a positive multidimensional space distance $M$ between the feature space and the generating space, so we can classify the data in the target space. The spatial distance is formed in different target classifications.

The first generator network is also satisfied for formula (11), and we define the generator network loss $L_{G}$ as follows:

$$
L_{G}=\min _{M} \sum_{\left(x_{i}, x_{j}\right) \in S}\left\|s_{i}-s_{j}\right\|_{M}^{2} .
$$

The error of the generator network is satisfied, which is to satisfy

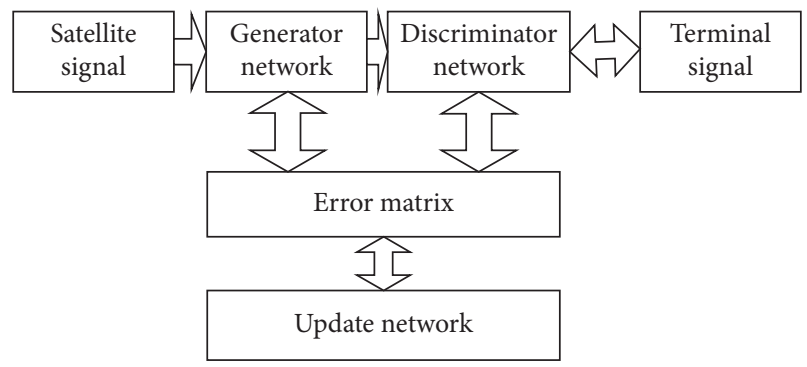

FIGURE 10: GAN network model information processing flow for the satellite signal.

$$
\min _{M} \sum_{\left(x_{i}, x_{j}\right) \in D}\left\|s_{i}-s_{j}\right\|_{M}^{2} \geq 1 .
$$

The spatial distance can be expressed as follows:

$$
L_{G}=\min _{M} \sqrt{\left(s_{i}-s_{j}\right)^{T} M\left(s_{i}-s_{j}\right)} .
$$

Further simplification is as follows:

$$
L_{G}=\min _{M} \sqrt{\left(s_{i}-s_{j}\right)^{T} W W^{T}\left(s_{i}-s_{j}\right)},
$$

where $W$ is the weight matrix of the GAN network. 


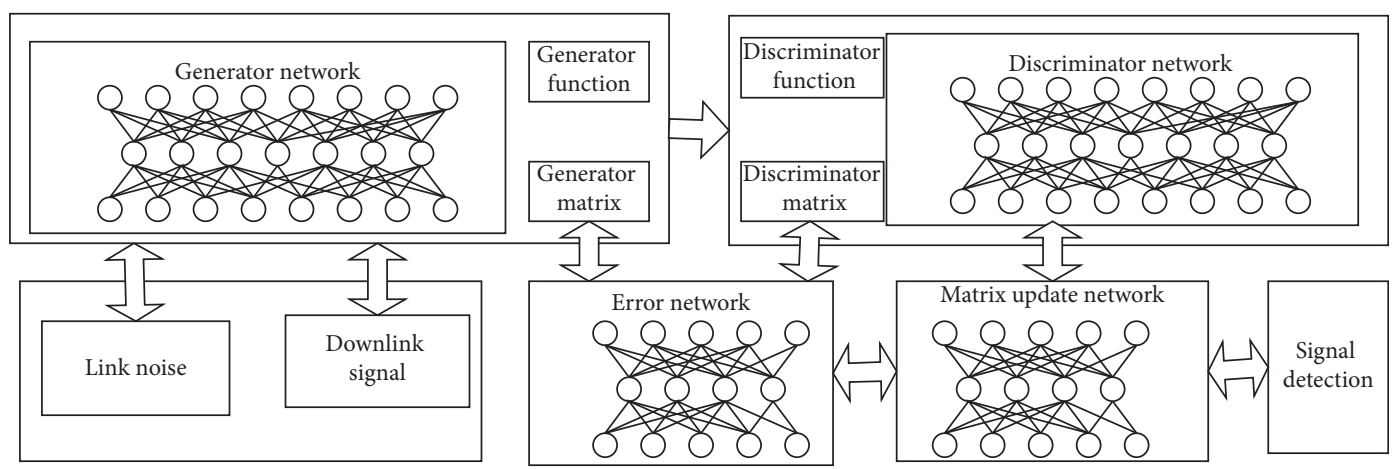

Figure 11: Signal processing flow based on the adversarial network.

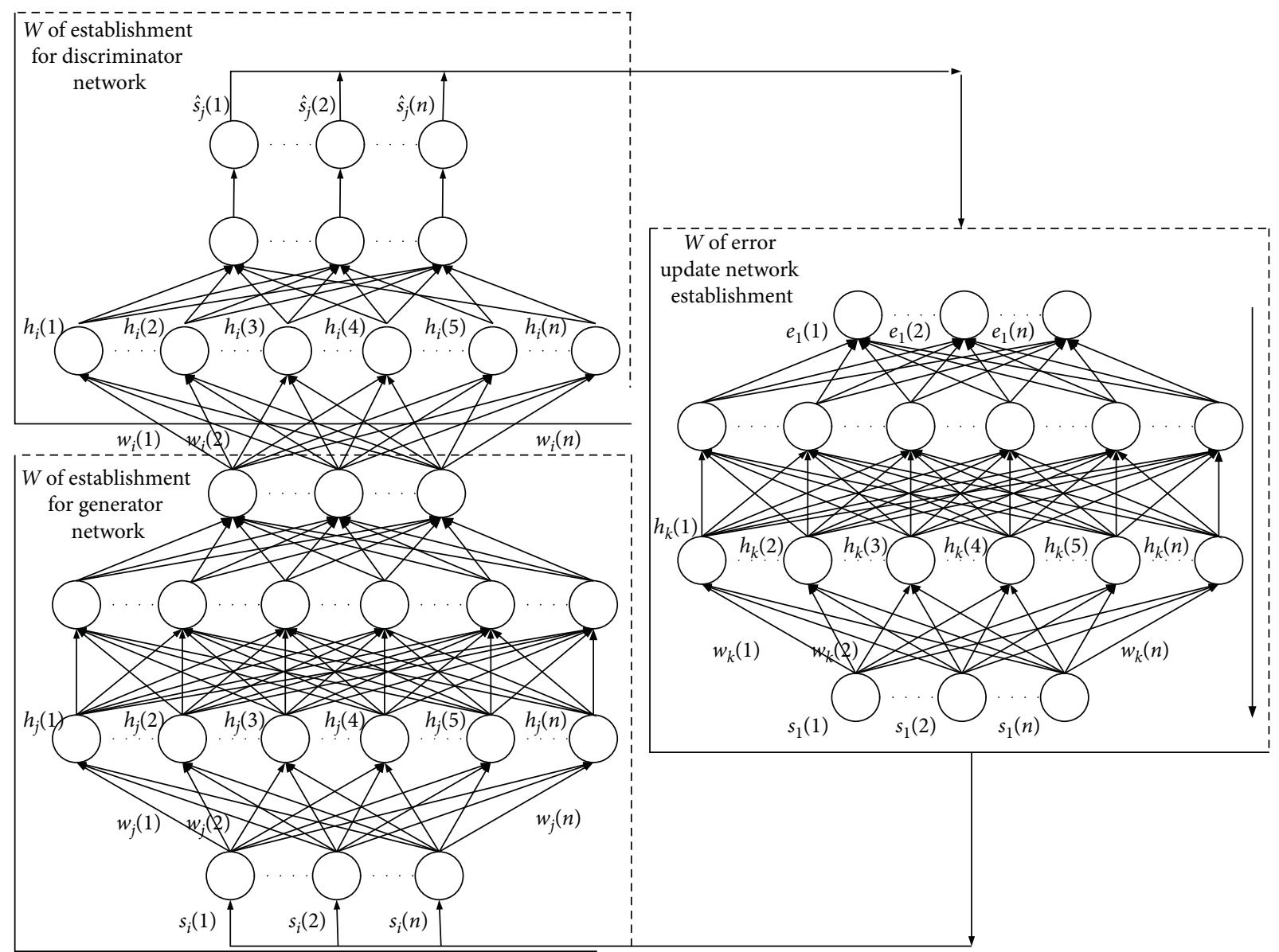

FIgURE 12: Flow for weight $W$ establishment of the GAN network.

3.3. Establishment for Discriminator Network. Establish the extraction direction of the discriminator feature space, and we could obtain as follows:

$$
L_{\mathrm{GAN}}=L_{G}-\alpha L_{D},
$$

where $L_{G}$ represents the cost function for the generator network and $L_{D}$ represents the cost function for the discriminator network. We also define the elimination coefficients $\alpha$ for generator and adversarial networks:

$$
\alpha=\frac{\operatorname{tr}\left(W^{T} S L_{s} S^{T} W\right)}{\operatorname{tr}\left(W^{T} S L_{D} S^{T} W\right)} .
$$

Further, the weight can be obtained as follows through GAN network: 


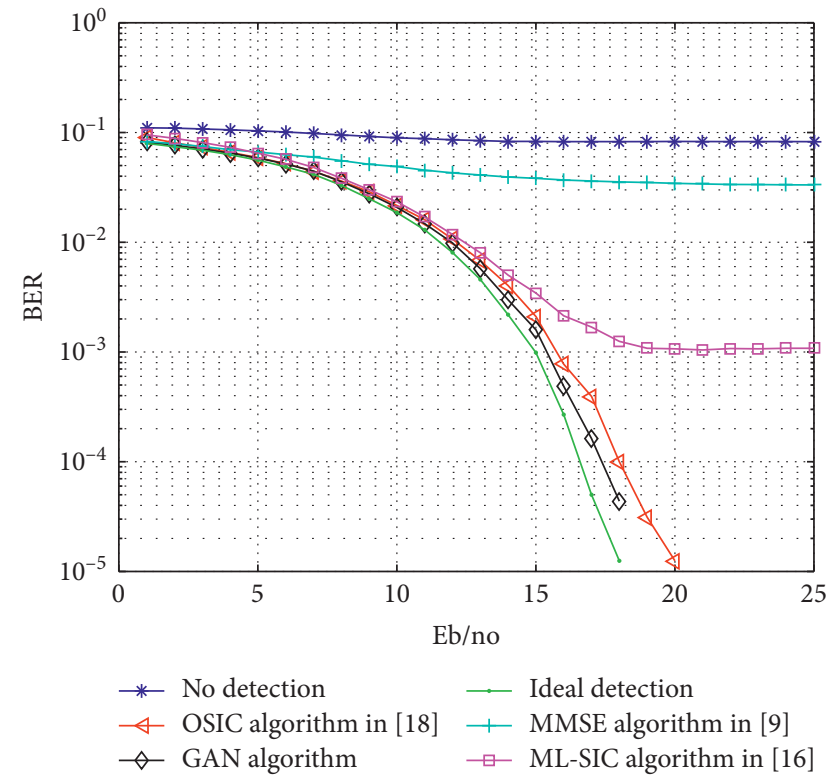

FIGURE 13: BER performance of QPSK signal in the rural environment channel model.

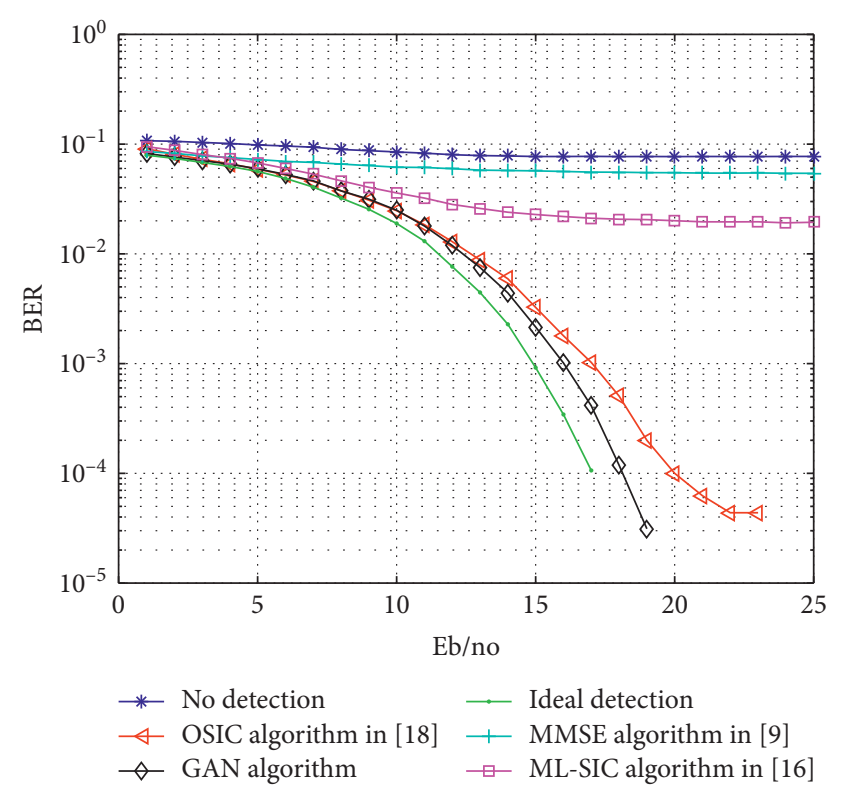

FIGURE 14: BER performance of QPSK in the urban environment channel model.

$$
W=\underset{W^{T} W=1}{\arg \min } \operatorname{tr}\left(W^{T} S\left(L_{\mathrm{GAN}}\right) S^{T} W\right)
$$

3.4. Establishment for Error Update Network. Furthermore, we establish an error network as follows:

$$
W=\underset{W^{T} W=1}{\arg \min } \operatorname{tr}\left(W^{T} S\left(L_{s}-\alpha L_{D}\right) S^{T} W\right),
$$

where $\operatorname{tr}(\cdot)$ represents the operation of taking matrix traces, in which $\alpha$ is defined as the distance measurement parameter

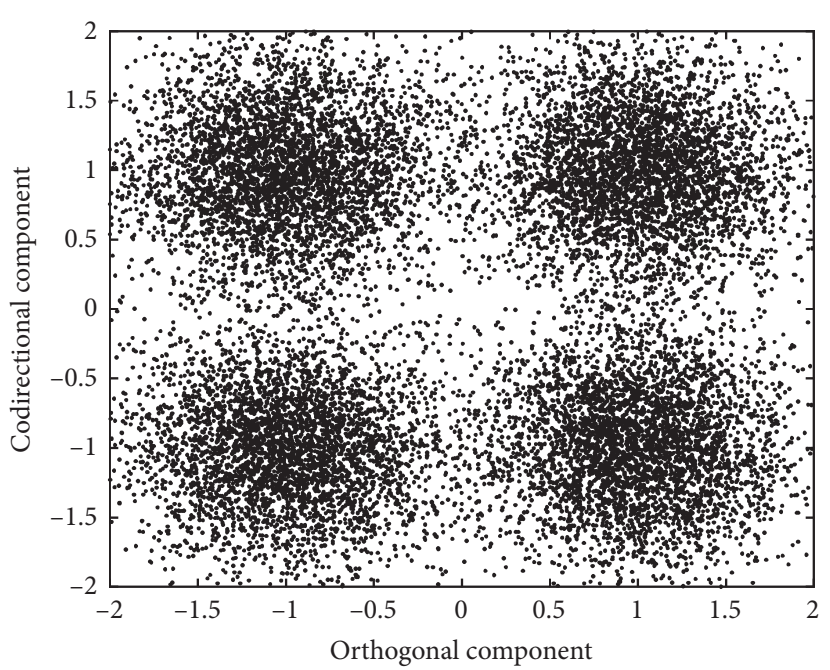

FIGURE 15: QPSK signal constellation (after the MMSE algorithm in [9]).

of the two adversarial matrix feature spaces, expressed as follows.

Through extensibility analysis, further calculations can be obtained:

$$
\begin{aligned}
W & =\underset{W^{T} W=1}{\arg \min } \operatorname{tr}\left(W^{T} S\left(L_{s}-\alpha L_{D}\right) S^{T} W\right) \\
& =\underset{W^{T} W=1}{\arg \min } \operatorname{tr}\left(W^{T} S\left(U \sum U^{T}\right) S^{T} W\right) \\
& =\underset{W^{T} W=1}{\arg \min } \operatorname{tr}\left(W^{T} S\left(U \sum^{1 / 2} \sum^{1 / 2} U^{T}\right) S^{T} W\right) \\
& =\underset{W^{T} W=1}{\arg \min } \operatorname{tr}\left(W^{T} S\left(U_{\Sigma} U^{T}\right) S^{T} W\right) .
\end{aligned}
$$

To further obtain an optimized representation of the weights for GAN network,

$$
W=\underset{W^{T} W=1}{\arg \min }\left\|W^{T} S U\right\|_{F}^{2} .
$$

Figure 12 gives the flow for weight $W$ establishment of the GAN network, which shows the three processing model implemented with the GAN network through the weight $\left\{W_{i j k}(n)\right\}=\left\{w_{i j k}(1), w_{i j k}(2), \ldots, w_{i j k}(n)\right\}$.

The meaning of the parameters in Figure 12 is as follows.

The weight of the GAN network includes the process of generator weight $w_{j}(n)$ transfer, the process of discriminator weight $w_{i}(n)$ transfer, and the process of error weight $w_{k}(n)$ update through the network.

$\left\{h_{i j k}(n)\right\}=\left\{h_{i j k}(1), h_{i j k}(2), \ldots, h_{i j k}(n)\right\}$ is defined as the activation function of the GAN network, including the generator network activation function $h_{j}(n)$, the discriminator network activation function $h_{i}(n)$, and the error updating network activation function $h_{k}(n)$.

$\left\{s_{i}, \widehat{s}_{j}\right\}=\left\{\left(s_{1}, \widehat{s}_{1}\right),\left(s_{2}, \widehat{s}_{2}\right), \ldots,\left(s_{i}, \widehat{s}_{j}\right)\right\}$ is defined as the generation set during the generation process given by formula (12). During this processing, $\left\{s_{i}\right\}=\left\{s_{1}, s_{2}, \ldots, s_{i}\right\}$ is 


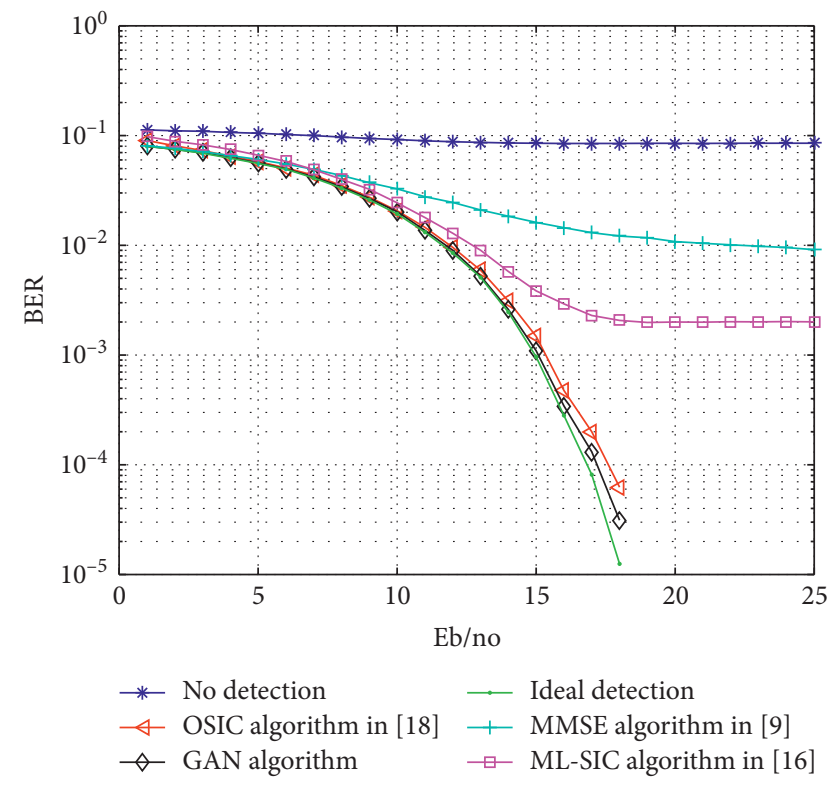

FIGURE 16: BER performance of QPSK signal in the wilderness environment channel model.

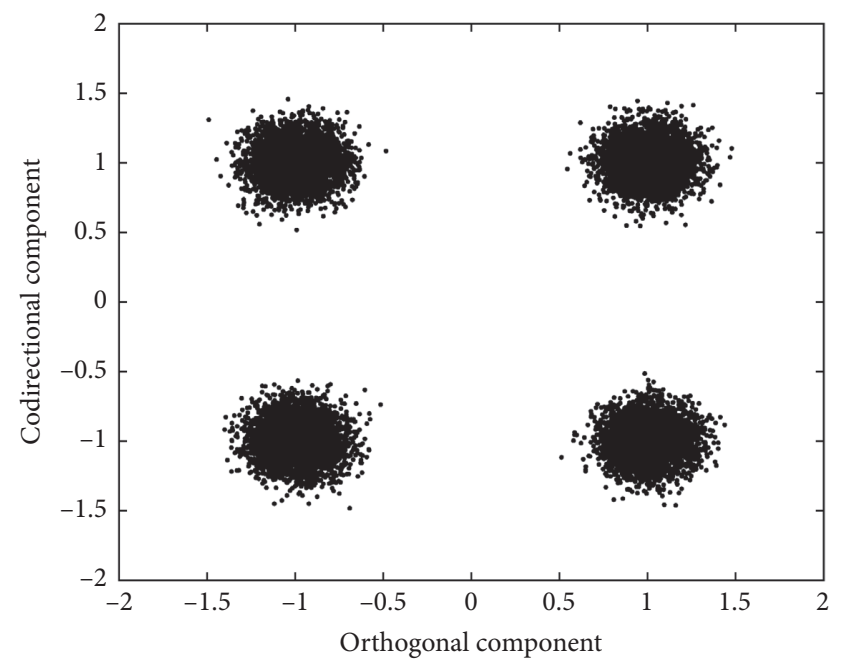

FIGURE 17: QPSK signal constellation (after the ML-SIC algorithm in [16]).

defined as the local data. $\left\{\widehat{s}_{j}\right\}=\left\{\widehat{s}_{1}, \widehat{s}_{2}, \ldots, \widehat{s}_{j}\right\}$ is defined as the generation set. $\left\{e_{j}(n)\right\}=\left\{e_{j}(1), e_{j}(2), \ldots, e_{j}(n)\right\}$ is the $j$ th error between the $j$ th generator network and the $j$ th discriminator network, which is produced by the processing between the generator network and the discriminator network.

The generator space $w_{j}(n)$ is established by optimizing the weights as shown in the generator part and then in the discriminator process given in formula (16). The discriminator space $w_{i}(n)$ is established as shown in the discriminator part. Then, the optimal weight $w_{k}(n)$ is obtained by updating the error network.

nonlinear processing and sparse coding introduce the nonlinearization to the learning target, especially due to the noise interference.. Regularization becomes complicated,

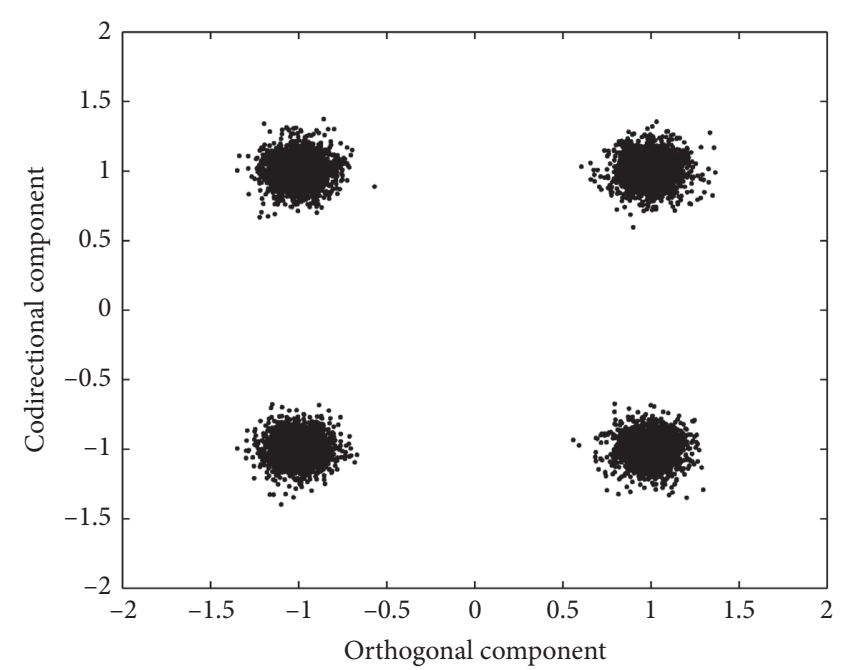

FIGURE 18: QPSK signal constellation (after the OSIC algorithm in [18]).

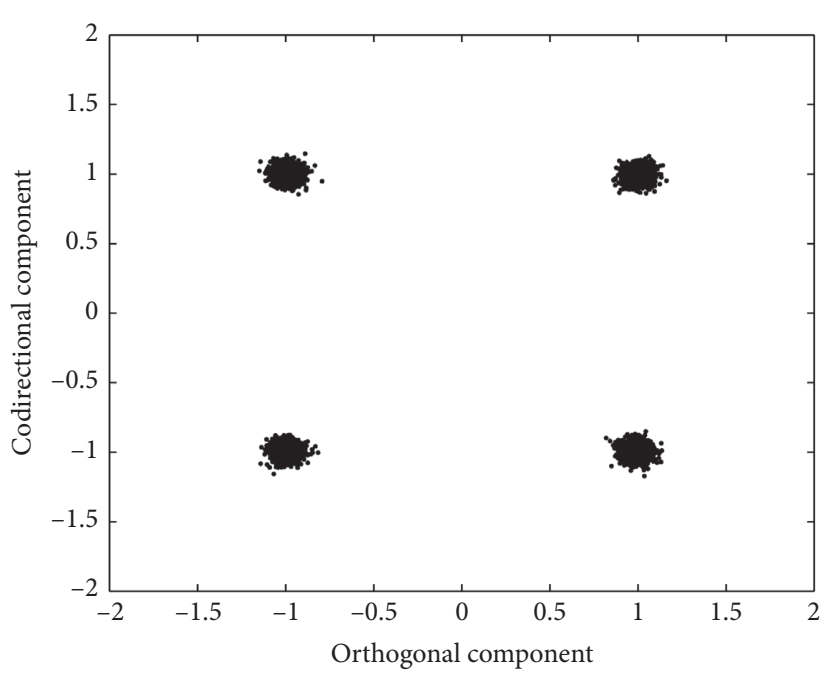

FIGURE 19: QPSK signal constellation (after the GAN algorithm proposed).

especially for the feature transformation of the projection space, and it is difficult to regularize in the feature space. Therefore, we use a simplified method to replace and reduce the impact of noise. The sparse distance loss function adopted can obtain better target features.

3.5. Process for GAN Network Optimization. Further, optimization is available as follows:

$$
\min _{W} \sum_{\left(x_{i}, x_{j}\right) \in D}\|Y-W S\|_{F}^{2}+\alpha \operatorname{rank}\left(W S U_{\Sigma}\right),
$$

where we define as

$$
\text { s.t. }\|W\|_{2}^{2} \leq 1 .
$$

Further, $\operatorname{rank}(\cdot)$ is the representation matrix which takes a rank operation. $W X U_{\Sigma}$ is to identify feature limitations for 


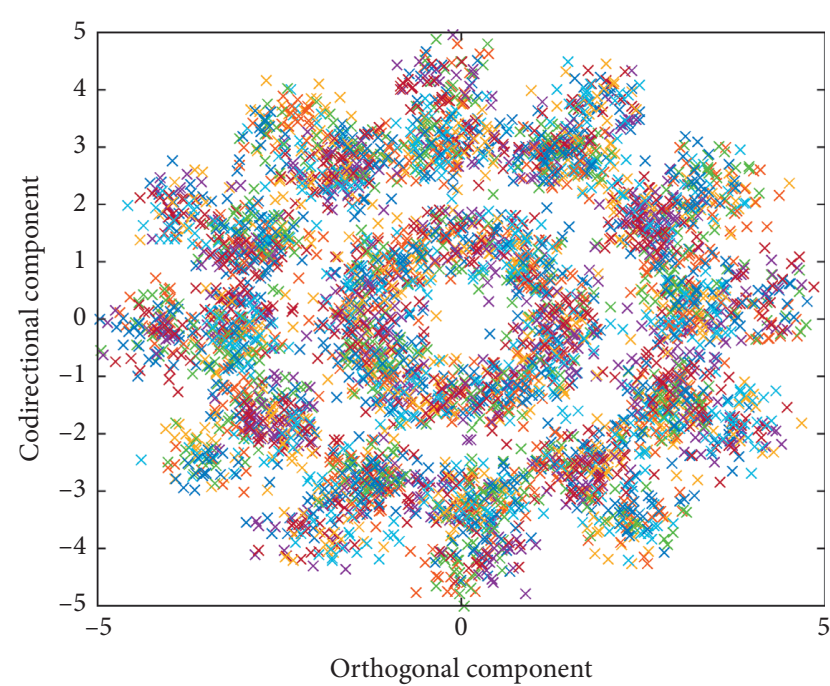

FIGURE 20: 16QAM signal constellation (undetected multisatellite signal).

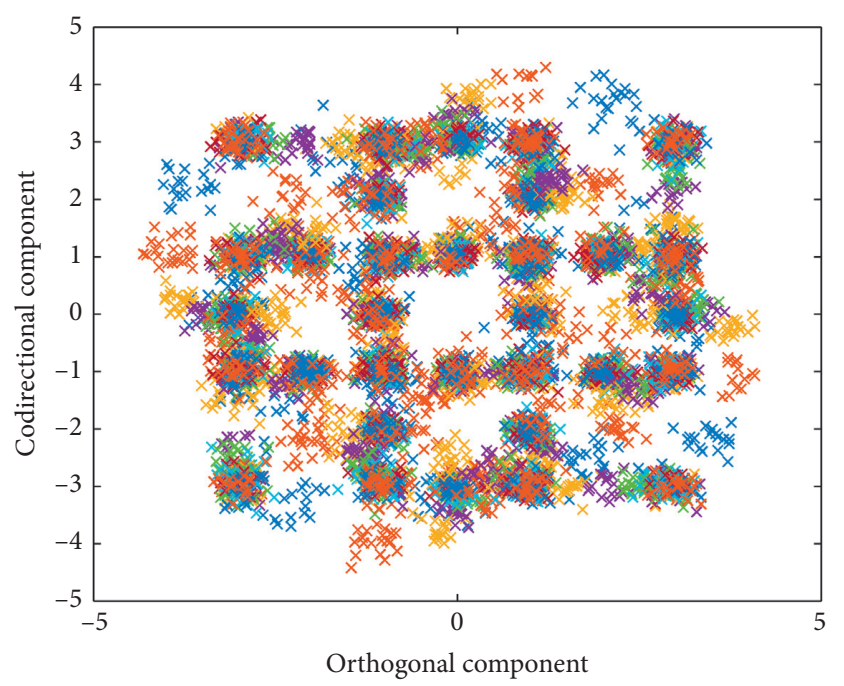

FIGURE 21: 16QAM signal constellation (detected with no generator network).

different user signals in the process of matrix confrontation. For example, the modulation mode of the received data detection signal can be QPSK, BPSK, 16QAM, etc.

Because rank minimization is an NP problem, it is necessary to obtain optimal convergence in the feature space. In particular, the loss function can be expressed by minimizing the rank of the matrix. By completing the operation of the minimum rank of the feature transformation matrix, the objective loss of the function is to obtain the optimal recognition. In order to improve the structural consistency of the cost function and reduce the influence of sampling interference, the cost loss function gives the low-rank optimization method.

Discussion: we should optimize the objective solution. Among them, $\|\cdot\|_{1}$ is considered as the modular matrix. The minimum optimization equation is established to solve $W$ as shown below:

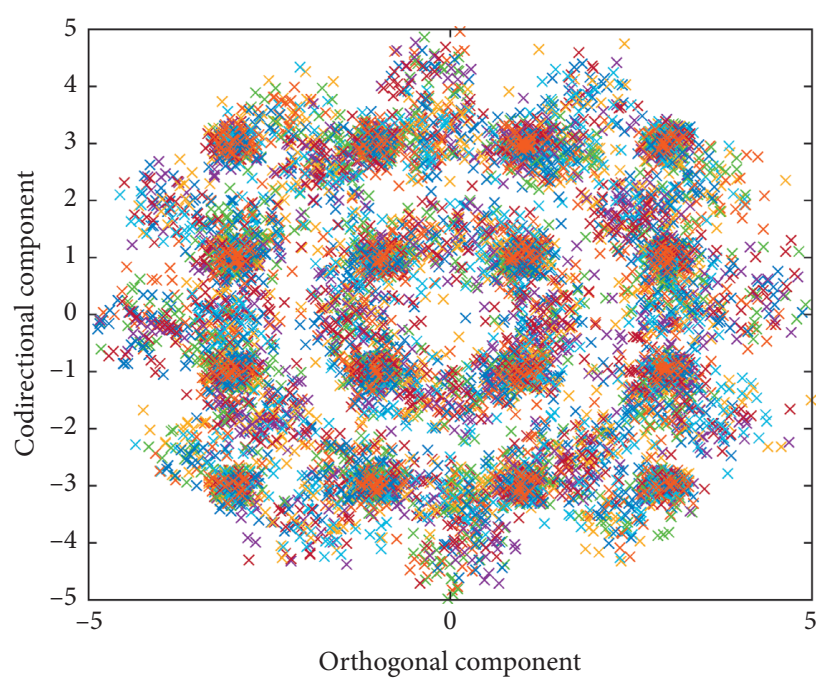

FIGURE 22: 16QAM signal constellation (detected with no error update network).

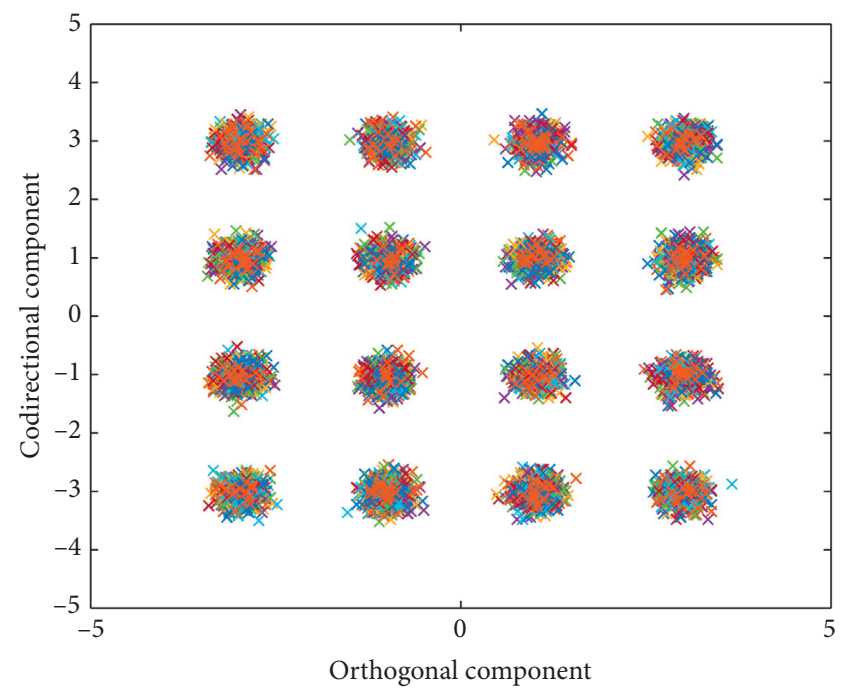

FIgURE 23: 16QAM signal constellation (detected with GAN network).

$$
\begin{aligned}
J & =\|Y\|_{1}+\frac{\mu}{2}\left\|W^{T} S U_{\Sigma}\right\|_{1}^{2}, \\
\text { s.t. } \quad W^{T} W & =I .
\end{aligned}
$$

To construct an optimization method, convex theory seeks optimization.

\section{Experimental Classification Results and Analysis}

In order to verify the detection performance of the proposed GAN algorithm, the average altitude for the satellite is defined as $1450 \mathrm{~km}$, the number of low-orbit satellites is defined as 10 , and the spot beams were defined as 7 . The parameters for low-orbit satellites are set in accordance with the wilderness model, rural model, and the urban model 


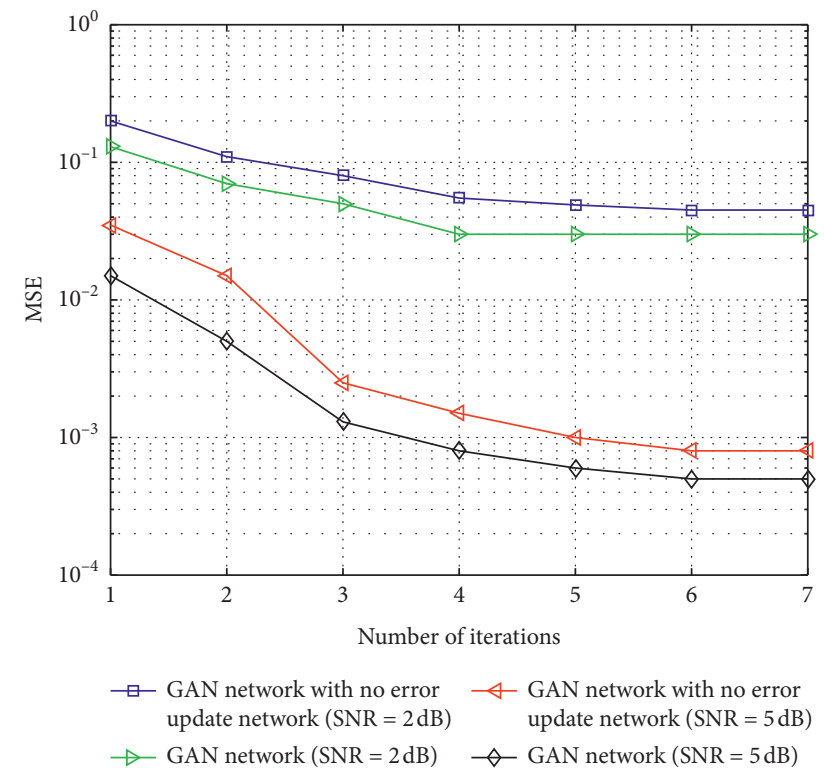

FIGURE 24: Analysis of stability based on the proposed algorithm.

which are established in the simulation scenarios. Define the maximum working elevation angle of satellite as $35^{\circ}$. Define the downlink for low-orbit satellite transmission bit rate as $160 \mathrm{Mbit} / \mathrm{s}$. We set that the cyclic prefix is greater than the spread delay. We also set the modulation as QPSK.

4.1. BER Analysis. The proposed adversarial network algorithm can be verified by Matlab. The maximum frequency deviation range allowed as the interval of subcarrier. Figures 13-15 show the bit error rate curves under different rural scenarios of low-orbit satellite downlinks, when ground users receive multiple satellite signals. The related algorithms include the frequency domain equalization algorithm based on the MMSE criterion proposed in [9], the ML-SIC algorithm criterion proposed in [16], the iterative order SIC algorithm proposed in [18], and the generative adversarial network proposed in this paper.

The conditions in Figures 13, 14 and 16 assume that each subchannel is independent. It can be obtained from the BER curve that the curve without the elimination is larger. Due to the large frequency offset interference, with the increase in SNR, the BER performance is not increasing. The MMSE algorithm can eliminate part of the interference. The ML-SIC algorithm has better performance, and it can eliminate the interference caused by phase rotation and can suppress the interference introduced by noise. Compared with the SIC algorithm based on the MMSE estimation criterion, the frequency offset preelimination based on the adversarial network algorithm improves the frequency domain frequency offset cancellation performance, especially when the frequency offset is large, the performance of frequency offset cancellation will be significantly improved.
4.2. Constellation Analysis. The downlink multisatellite detection based on the generative adversarial network can be obtained through the simulation of QPSK constellation, which is still selected as the signal mapping. Assuming $\mathrm{SNR}=15 \mathrm{~dB}$, the downlink received signal is defined in an urban environment. Figure 15 shows the signal constellation after the MMSE algorithm in [9]. Figure 17 shows the signal constellation after the ML-SIC algorithm in [16]. Figure 18 shows the signal constellation after the OSIC algorithm in [18]. Figure 19 shows the signal constellation after the GAN algorithm proposed in this paper. Compared with the constellation simulation used in Figures 17-19, the adversarial network can better suppress the interference introduced by the larger carrier frequency offset and improve the accuracy of downlink signal cancellation.

4.3. Integrity Analysis. In order to verify the integrity of the three networks more effectively and reflect the differentiation of simulation, we use the simulation of 16QAM constellation for the downlink multisatellite detection. Assuming $\mathrm{SNR}=10 \mathrm{~dB}$, the downlink received signal is defined in an urban environment.

Figure 20 shows the signal constellation with no detection. Figure 21 shows the signal constellation after detection with no generator network. Figure 22 shows the signal constellation after detection with no error update network. Figure 23 shows the proposed GAN network. Compared with the constellation simulation used, the adversarial network with three networks, including generator network, discriminator network, and error update network, can better suppress the interference and improve the accuracy of downlink signal cancellation.

4.4. Convergence Analysis. Figure 24 gives the analysis of stability based on the proposed algorithm. The stability of the proposed algorithm is obtained through the convergence learning curve. We research whether the MSE convergence performance of the system combined with the error update network. By defining $S N R=5 \mathrm{~dB}$ and $S N R=2 \mathrm{~dB}$, respectively, we get the simulation performance. After 5 iterations, the MSE has reached $10 e-3$. Under the same conditions, the convergence speed of no error update is slower, and this is because the error update could improve the convergence learning performance.

\section{Conclusion}

In this paper, we have proposed the method for satellite downlink signal detection based on the GAN network. We establish the generator network and adversarial network, respectively. The generator network is established with the local generator of virtual satellite signals, and the adversarial network is established for high-precision signal detection. And we also have established the error network with the error signal from satellite downlink. Then we form an adaptive matrix weight adjustment. Compared with traditional shallow networks, such as MMSE, ML-SIC 
algorithms, and iterative algorithms, under the same signalto-noise ratio, the performance is improved by $5 \mathrm{~dB}$.

\section{Data Availability}

The data used to support the findings of this work are available from the corresponding author upon request.

\section{Conflicts of Interest}

The authors declare that they have no conflicts of interest.

\section{Authors' Contributions}

Qingyang Guan and Shuang Wu contributed equally to this work.

\section{Acknowledgments}

This work was supported by the National Natural Science Foundation of China (no. 61501306), Scientific Research Initiation Funds for the Doctoral Program of Xi'an International University (Grant nos. XAIU2019002 and XAIU2018070102), General Project of Science and Technology Department of Shaanxi Province (Grant no. 2020JM638), and the Natural Science Foundation of Liaoning Province of China (no. 2015020026).

\section{References}

[1] S. N. Tran and A. S. d'Avila Garcez, "Deep logic networks: inserting and extracting knowledge from deep belief networks," IEEE Transactions on Neural Networks and Learning Systems, vol. 29, no. 2, pp. 246-258, 2018.

[2] G. Hinton, L. Deng, D. Yu et al., "Deep neural networks for acoustic modeling in speech recognition: the shared views of four research groups," IEEE Signal Processing Magazine, vol. 29, no. 6, pp. 82-97, 2012.

[3] N. C. Luong, D. Thai Hoang, and S. Gong, "Applications of deep reinforcement learning in communications and networking: a survey," IEEE Communications Surveys \& Tutorials, vol. 21, no. 4, pp. 3133-3174, 2019.

[4] E. J. Candès and T. Tao, "Near-optimal signal recovery from random projections: universal encoding strategies?" IEEE Transactions on Information Theory, vol. 52, no. 12, pp. 5406-5425, 2006.

[5] E. Candes and T. Tao, "The dantzig selector: statistical estimation when $p$ is much larger than $n$," The Annals of Statistics, vol. 35, no. 6, pp. 2313-2351, 2007.

[6] M. M. Hyder and K. Mahata, "Direction-of-arrival estimation using a mixed $l_{2,0}$ norm approximation," IEEE Transactions on Signal Processing, vol. 58, no. 9, pp. 4646-4655, 2010.

[7] R. Prasad, C. R. Murthy, and B. D. Rao, "Joint approximately sparse channel estimation and data detection in OFDM systems using sparse Bayesian learning," IEEE Transactions on Signal Processing, vol. 62, no. 14, pp. 3591-3603, 2014.

[8] Z. Chen, L.-Y. Duan, S. Wang et al., "Toward knowledge as a service over networks: a deep learning model communication paradigm," IEEE Journal on Selected Areas in Communications, vol. 37, no. 6, pp. 1349-1363, 2019.

[9] S. Gaur and M. Ingram, "Simple MMSE interference suppression for real and rate-1/2 complex orthogonal space-time block codes," IEEE Transactions on Wireless Communications, vol. 7, no. 8, pp. 2901-2904, 2008.

[10] H. Sampath and A. Paulraj, "Linear precoding for space-time coded systems with known fading correlations," IEEE Communications Letters, vol. 6, no. 6, pp. 239-241, 2002.

[11] A. Scaglione, P. Stoica, S. Barbarossa, G. B. Giannakis, and H. Sampath, "Optimal designs for space-time linear precoders and decoders," IEEE Transactions on Signal Processing, vol. 50, no. 5, pp. 1051-1064, 2002.

[12] H. Giannakis, P. Stoica, and A. Paulraj, "Generalized linear precoder and decoder design for MIMO channels using the weighted MMSE criterion," IEEE Transactions on Communications, vol. 49, no. 12, pp. 2198-2206, 2001.

[13] C. Studer, S. Fateh, and D. Seethaler, "ASIC implementation of soft-input soft-output MIMO detection using MMSE parallel interference cancellation," IEEE Journal of Solid-State Circuits, vol. 46, no. 7, pp. 1754-1765, 2011.

[14] M. Cirkic and E. Larsson, "SUMIS: near-optimal soft-in soft-out MIMO detection with low and fixed complexity," IEEE Transactions on Signal Processing, vol. 62, no. 12, pp. 3084-3097, 2014.

[15] S. K. Mohammed and E. G. Larsson, "Per-antenna constant envelope precoding for large multi-user MIMO systems," IEEE Transactions on Communications, vol. 61, no. 3, pp. 1059-1071, 2013.

[16] M. B. Çelebi and H. Arslan, "Theoretical analysis of the coexistence of LTE-A signals and design of an ML-SIC receiver," IEEE Transactions on Wireless Communications, vol. 14, no. 8 , pp. 4626-4639, 2015.

[17] F. Rusek, D. Persson, B. K. Lau, E. Larsson, and T. Marzetta, "Scaling up MIMO: opportunities and challenges with very large arrays," IEEE Signal Processing Magazine, vol. 30, no. 1, pp. 40-60, 2013.

[18] J. Ahn, B. Shim, and K. B. Lee, "Sparsity-aware ordered successive interference cancellation for massive machine-type communications," IEEE Wireless Communications Letters, vol. 7, no. 1, pp. 134-137, 2018.

[19] P. Ghamisi, M. Dalla Mura, and J. A. Benediktsson, “A survey on spectral-spatial classification techniques based on attribute profiles," IEEE Transactions on Geoscience and Remote Sensing, vol. 53, no. 5, pp. 2335-2353, 2015.

[20] Y. Gu, J. Chanussot, X. Jia, and J. A. Benediktsson, "Multiple kernel learning for hyperspectral image classification: a review," IEEE Transactions on Geoscience and Remote Sensing, vol. 55, no. 11, pp. 6547-6565, 2017.

[21] Y. Yuan, J. Lin, and Q. Wang, "Hyperspectral image classification via multitask joint sparse representation and stepwise MRF optimization," IEEE Transactions on Cybernetics, vol. 46, no. 12, pp. 2966-2977, 2016.

[22] Q. Wang, Z. Meng, and X. Li, "Locality adaptive discriminant analysis for spectral-spatial classification of hyperspectral images," IEEE Geoscience and Remote Sensing Letters, vol. 14, no. 11, pp. 2077-2081, 2017.

[23] Y. Bengio, A. Courville, and P. Vincent, "Representation learning: a review and new perspectives," IEEE Transactions on Pattern Analysis and Machine Intelligence, vol. 35, no. 8, pp. 1798-1828, 2013.

[24] X. Yuan, P. He, Q. Zhu, and X. Li, “Adversarial examples: attacks and defenses for deep learning," IEEE Transactions on Neural Networks and Learning Systems, vol. 30, no. 9, pp. 2805-2824, 2019.

[25] L. Zhang, L. Zhang, and B. Du, "Deep learning for remote sensing data: a technical tutorial on the state of the art," IEEE Geoscience and Remote Sensing Magazine, vol. 4, no. 2, pp. 22-40, 2016. 
[26] Y. Chen, Z. Lin, X. Zhao, G. Wang, and Y. Gu, "Deep learning-based classification of hyperspectral data," IEEE Journal of Selected Topics in Applied Earth Observations and Remote Sensing, vol. 7, no. 6, pp. 2094-2107, 2014.

[27] Y. Chen, X. Zhao, and X. Jia, "Spectral-spatial classification of hyperspectral data based on deep belief network," IEEE Journal of Selected Topics in Applied Earth Observations and Remote Sensing, vol. 8, no. 6, pp. 1-12, 2015.

[28] P. Zhong, Z. Gong, S. Li, and C.-B. Schönlieb, "Learning to diversify deep belief networks for hyperspectral image classification," IEEE Transactions on Geoscience and Remote Sensing, vol. 55, no. 6, pp. 3516-3530, 2017.

[29] W. Hu, Y. Huang, L. Wei, F. Zhang, and H. Li, “Deep convolutional neural networks for hyper spectral image classification," Journal of Sensors, vol. 2015, Article ID 258619, 2015.

[30] W. Li, G. Wu, F. Zhang, and Q. Du, "Hyperspectral image classification using deep pixel-pair features," IEEE Transactions on Geoscience and Remote Sensing, vol. 55, no. 2, pp. 844-853, 2017.

[31] W. Shao and S. Du, "Spectral-spatial feature extraction for hyper-spectral image classification: a dimension reduction and deep learning approach," IEEE Transactions on Geoscience and Remote Sensing, vol. 54, no. 8, pp. 4544-4554, 2016.

[32] J. Yue, W. Zhao, S. Mao, and H. Liu, "Spectral-spatial classification of hyperspectral images using deep convolutional neural networks," Remote Sensing Letters, vol. 6, no. 6, pp. 468-477, 2015.

[33] Y. Chen, H. Jiang, C. Li, X. Jia, and P. Ghamisi, "Deep feature extraction and classification of hyperspectral images based on convolutional neural networks," IEEE Transactions on Geoscience and Remote Sensing, vol. 54, no. 10, pp. 6232-6251, 2016.

[34] Y. Li, H. Zhang, and Q. Shen, "Spectral-spatial classification of hyperspectral imagery with 3D convolutional neural network," Remote Sensing, vol. 9, no. 1, p. 67, 2017.

[35] H. Liang and Q. Li, "Hyperspectral imagery classification using sparse representations of convolutional neural network features," Remote Sensing, vol. 8, no. 2, p. 99, 2016.

[36] Y. Chen, L. Zhu, P. Ghamisi, X. Jia, G. Li, and L. Tang, "Hyperspectral images classification with gabor filtering and convolutional neural network," IEEE Geoscience and Remote Sensing Letters, vol. 14, no. 12, pp. 2355-2359, 2017.

[37] C. Zhang, X. Yang, Y. Tang, and W. Zhang, "Learning to generate radar image sequences using two-stage generative adversarial networks," IEEE Geoscience and Remote Sensing Letters, vol. 17, no. 3, pp. 401-405, 2020.

[38] C. Wang, C. Xu, X. Yao, and D. Tao, "Evolutionary generative adversarial networks," IEEE Transactions on Evolutionary Computation, vol. 23, no. 6, pp. 921-934, 2019.

[39] Y. Pang, J. Xie, and X. Li, "Visual haze removal by a unified generative adversarial network," IEEE Transactions on Circuits and Systems for Video Technology, vol. 29, no. 11, pp. 3211-3221, 2019.

[40] X. Chen, C. Xu, X. Yang, L. Song, and D. Tao, "Gated-GAN: adversarial gated networks for multi-collection style transfer," IEEE Transactions on Image Processing, vol. 28, no. 2, pp. 546-560, 2019.

[41] A. Creswell, T. White, V. Dumou, and K. Lin, "Generative adversarial networks: an overview," IEEE Signal Processing Society, vol. 35, no. 1, pp. 53-65, 2017.

[42] Y. Sun, J. Tang, X. Shu, Z. Sun, and M. Tistarelli, "Facial age synthesis with label distribution-guided generative adversarial network," IEEE Transactions on Information Forensics and Security, vol. 15, pp. 2679-2691, 2020.

[43] H. Yu, Z. Li, G. Zhang, P. Liu, and J. Wang, "Extracting and predicting taxi hotspots in spatiotemporal dimensions using conditional generative adversarial neural networks," IEEE Transactions on Vehicular Technology, vol. 69, no. 4, pp. 3680-3692, 2020.

[44] Z. Yuan, H. Li, J. Liu, and J. Luo, "Multiview scene image inpainting based on conditional generative adversarial networks," IEEE Transactions on Intelligent Vehicles, vol. 5, no. 2, pp. 314-323, 2020.

[45] C. Jia, X. Zhang, S. Wang, S. Wang, and S. Ma, "Light field image compression using generative adversarial networkbased view synthesis," IEEE Journal on Emerging and Selected Topics in Circuits and Systems, vol. 9, no. 1, pp. 177-189, 2019.

[46] M. Mardani, E. Gong, J. Y. Cheng et al., "Deep generative adversarial neural networks for compressive sensing MRI," IEEE Transactions on Medical Imaging, vol. 38, no. 1, pp. 167-179, 2019.

[47] Y. Lyu, Z. Han, J. Zhong, C. Li, and Z. Liu, "A generic anomaly detection of catenary support components based on generative adversarial networks," IEEE Transactions on Instrumentation and Measurement, vol. 69, no. 5, pp. 2439-2448, 2020.

[48] H. Li, S. Zhou, W. Yuan, J. Li, and H. Leung, "Adversarialexample attacks toward android malware detection system," IEEE Systems Journal, vol. 14, no. 1, pp. 653-656, 2020.

[49] X. Xia, R. Togneri, F. Sohel, and D. Huang, "Auxiliary classifier generative adversarial network with soft labels in imbalanced acoustic event detection," IEEE Transactions on Multimedia, vol. 21, no. 6, pp. 1359-1371, 2019.

[50] M. Zhang, M. Gong, Y. Mao, J. Li, and Y. Wu, "Unsupervised feature extraction in hyperspectral images based on was serstein generative adversarial network," IEEE Transactions on Geoscience and Remote Sensing, vol. 57, no. 5, pp. 26692688, 2019.

[51] Q. Xuan, Z. Chen, Y. Liu, H. Huang, G. Bao, and D. Zhang, "Multiview generative adversarial network and its application in pearl classification," IEEE Transactions on Industrial Electronics, vol. 66, no. 10, pp. 8244-8252, 2019. 